\title{
Bound Water Structure on the Surfaces of Usnea antarctica as Observed by NMR and Sorption Isotherm
}

\author{
H. HarańczyK ${ }^{a, *}$, A. PietrzyK ${ }^{b}$, A. Leja ${ }^{a}$ And M.A. Olech ${ }^{c}$ \\ ${ }^{a}$ Institute of Physics, Jagiellonian University \\ Reymonta 4, 30-059 Kraków, Poland \\ ${ }^{b}$ Institute of Nuclear Physics, Polish Academy of Sciences \\ Radzikowskiego 152, 31-342 Kraków, Poland \\ ${ }^{c}$ Institute of Botany, Jagiellonian University \\ Kopernika 27, 31-501 Kraków, Poland
}

\begin{abstract}
Hydration courses and proton free induction decays are recorded at $30 \mathrm{MHz}$ for Usnea antarctica thalli hydrated from gaseous phase. NMR data combined with gravimetry allow one to distinguish two fractions of tightly bound water, and loosely bound/free water pool. No water fraction "sealed" in thallus structures is present in $U$. antarctica.
\end{abstract}

PACS numbers: 82.56.Na, 87.16.Dg

\section{Introduction}

Antarctic lichens are extremophilic organisms very resistant on desiccation stress and cold [1-3]. They perform active photosynthesis below $0^{\circ} \mathrm{C}[4,5]$ and below ice nucleation of their cellular fluids [6]. An important mechanism of freezing resistance is the ice crystallite growth in extracellular spaces accompanied by simultaneous drastic decrease in hydration of intracellular spaces, thus the explanation of the molecular mechanisms of drought resistance and the mechanisms of cold resistance may be common. During acute desiccation stress lichen thallus is covered by single or less than monolayer of water [7], may hydrate from gaseous phase to the level allowing the initiation of photosynthesis [7, 8], and reversibly dehydrates below the two-dimensional percolation threshold of water [7]. Therefore, the knowledge on bound water structure (organization) may help in understanding of molecular mechanisms of the metabolic activity recovery during rehydration.

*corresponding author; e-mail: hubert.haranczyk@uj.edu.pl 
In our experiment we focused on water ordering, the number and distribution of water binding sites, sequence and kinetics of their saturation, as well as the formation of tightly and loosely bound water fractions at different steps of hydration process in Antarctic extremophilic fruticose lichen Usnea antarctica.

\section{Materials and methods}

U. antarctica thalli were collected in Maritime Antarctic, Antarctic Peninsula, King George Island, Polish Antarctic H. Arctowski Station neighbourhood during Antarctic summer [9]. Thalli were stored at room temperature in air-dry state (hydration level $\Delta m / m_{0}=0.10$ ). As a measure of thallus hydration, the relative mass increase, $\Delta m / m_{0}$, where $\Delta m$ is a mass increase, and $m_{0}$ is dry mass. The air-dry thalli were then chopped and placed in NMR tubes.

Before the hydration courses the samples were stabilized in the atmosphere over silica gel (relative humidity, $p / p_{0}=0 \%$ ), reaching the hydration level equal to $\Delta m / m_{0}=0.035$. The hydration courses were performed from the gaseous phase in the atmosphere over the surface of $\mathrm{H}_{3} \mathrm{PO}_{4}\left(p / p_{0}=9 \%\right)$, over the surfaces of supersaturated solutions of $\mathrm{CaCl}_{2}\left(p / p_{0}=32 \%\right), \mathrm{Na}_{2} \mathrm{Cr}_{2} \mathrm{O}_{7}\left(p / p_{0}=52 \%\right), \mathrm{Na}_{2} \mathrm{~S}_{2} \mathrm{O}_{3}$ $(76 \%), \mathrm{K}_{2} \mathrm{CrO}_{3}(88 \%), \mathrm{Na}_{2} \mathrm{SO}_{4}(93 \%)$, and over the water surface $\left(p / p_{0}=100 \%\right)$. After completing of the hydration courses, the dry mass of the thalli was determined after heating in oven at $70^{\circ} \mathrm{C}$ for $72 \mathrm{~h}$. Higher temperatures were not used as they may cause the decomposition of some organic constituents of thallus [10].

Proton free induction decays (FIDs) were recorded on high power WNS HB-65 relaxometer (Waterloo NMR Spectrometers, St. Agatha, Ontario, Canada) working at the resonance frequency $30 \mathrm{MHz}$ (at the field strength of $0.7 \mathrm{~T}$ ). The transmitter power was $400 \mathrm{~W}$; the pulse length $\pi / 2=1.35 \mu \mathrm{s}$. The data were acquired using Compuscope 2000 card of an IBM 80486 clone controlling the spectrometer and then averaged over 1000 accumulations. Repetition time was $2 \mathrm{~s}$. The NMR results were analyzed using the one-dimensional procedure of the CracSpin fitting program allowing the decomposition of the FID functions superimposed up to two Gaussian, seven exponentials, and two stretched exponential functions. The tests of the CracSpin effectivity were published elsewhere [11].

Hydration kinetics courses were performed on one different sample per given relative humidity. The NMR measurements were performed on five samples. Every sample covered a part of hydration range overlapped with others.

\section{Results}

The hydration kinetics of the $U$. antarctica thalli performed from the gaseous phase at the relative humidity (Fig. 1) was well fitted by the two-exponential function

$$
\Delta m / m_{0}=A_{0}^{\mathrm{h}}+A_{1}^{\mathrm{h}}\left[1-\exp \left(-t / t_{1}^{\mathrm{h}}\right)\right]+A_{2}^{\mathrm{h}}\left[1-\exp \left(-t / t_{2}^{\mathrm{h}}\right)\right],
$$

where $A_{0}^{\mathrm{h}}$ is the saturation level at incubation $\left(p / p_{0}=0 \%\right), A_{1}^{\mathrm{h}}$ and $A_{2}^{\mathrm{h}}$ are the saturation hydration levels for fast and slow component, $t_{1}^{\mathrm{h}}$ and $t_{2}^{\mathrm{h}}$ are hydration 


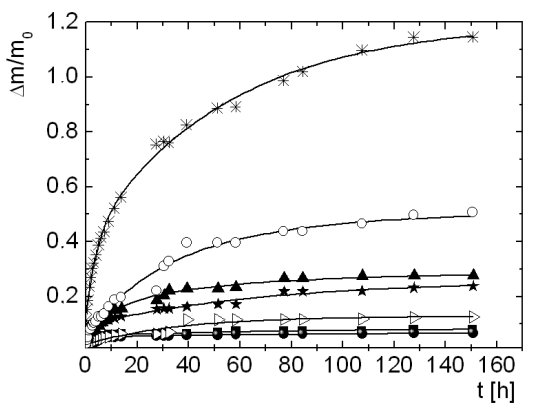

Fig. 1. Hydration kinetics of $U$. antarctica performed from the gaseous phase (target humidity $p / p_{0}=9 \%$ - shaded spheres, $p / p_{0}=32 \%$ - closed squares, $p / p_{0}=52 \%$ open triangles; $76 \%$ — closed pentagrame, $88 \%$ — closed triangles, 93 — open spheres, $p / p_{0}=100 \%$ - closed octagrame). The errors are within the plot symbols.

times for fast and slow component, respectively. For hydration courses performed to low humidity levels, the contribution of slow component was negligible. The values of parameters averaged over the full range of target humidities are equal to $A_{0}^{\mathrm{h}}=0.040 \pm 0.011, A_{1}^{\mathrm{h}}=0.087 \pm 0.028, t_{1}^{\mathrm{h}}=3.53 \pm 0.98 \mathrm{~h}$, and $t_{2}^{\mathrm{h}}=69.7 \pm 21.3 \mathrm{~h}$. The saturation level of the slow component, $A_{2}^{\mathrm{h}}$, increases gradually with increased humidity.

The saturation hydration level, $C^{\mathrm{h}}$, at given relative humidity, $p / p_{0}$, obtained from

$$
C^{\mathrm{h}}=A_{0}^{\mathrm{h}}+A_{1}^{\mathrm{h}}+A_{2}^{\mathrm{h}}
$$

was then taken for sorption isotherm.

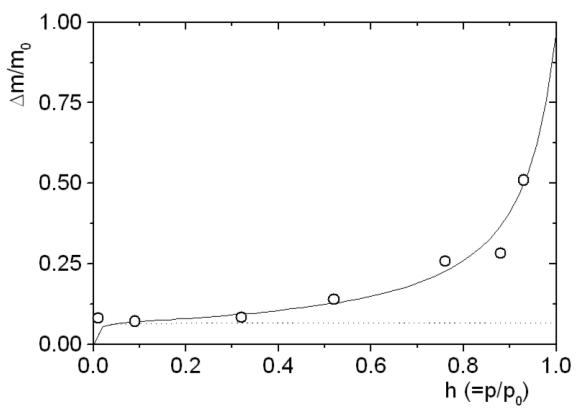

Fig. 2. Sorption isotherm for U. antarctica (solid line - fitted model, dotted line hydration of primary binding sites). The values of relative mass increase, $\Delta m / m_{0}$, are defined as the $C^{\text {h }}$ values from Eq. (2).

Sorption isotherm revealed sigmoidal form (Fig. 2) which is well fitted by the Dent model [12]: 


$$
C^{\mathrm{h}}(h)=\frac{\Delta M}{m_{0}} \frac{b_{1} h}{(1-b h)\left(1+b_{1} h-b h\right)},
$$

where $h=p / p_{0}$ is relative humidity expressed as ratio, $b$ is the relative coverage of the $n$-th water layer in units of the coverage of $(n-1)$-th layer, $S_{n+1} /\left.S_{n}\right|_{h=1}=b$, where $S_{i}$ is the population of $i$-th water layer, and for $U$. antarctica $b=0.913$; reciprocal of $b_{1}$ is a contribution of not populated primary water binding sites at $h=1, S_{0} /\left.N\right|_{h=1}=1 / b_{1}$, where $S_{0}$ is the number of water binding sites on the surface, for $U$. antarctica equal to 0.0076 ; and $\Delta M / m_{0}=0.0678$ is a mass of water saturating primary binding sites. The Dent model differentiates primary water binding sites to the surfaces of the thallus; and secondary water binding sites to water molecules already bound, or to the thallus water binding sites with lower strength of binding. Compared to other fruticose lichens, $U$. antarctica shows slightly elevated hydrophilicity of the thallus surfaces (higher $b_{1}$ value than for $U$. aurantiaco-atra), whereas the density of binding sites matches with that for other fruticose lichens [7].

Proton FID functions for $U$. antarctica thalli are well fitted by the superposition of one Gaussian component, with the amplitude $S$, coming from the solid matrix of thallus; and one, with the amplitude equal to $L_{1}$; or two, $L_{1}$ and $L_{2}$, exponential components (for the thalli hydrated in humidity level exceeding $h=0.10$ ) coming from water bound on the surfaces of thallus

$$
\mathrm{FID}(t)=S \exp \left[-\left(\frac{t}{T_{2 S}^{*}}\right)^{2}\right]+L_{1} \exp \left(-\frac{t}{T_{2 L_{1}}^{*}}\right)+L_{2} \exp \left(-\frac{t}{T_{2 L_{2}}^{*}}\right),
$$

where $T_{2 S}^{*}$ is proton relaxation time of solid component taken for $1 / \mathrm{e}$-value of Gaussian solid signal, and $T_{2 L_{1}}^{*}$ and $T_{2 L_{2}}^{*}$ are the relaxation times of proton liquid fractions $L_{1}$ and $L_{2}$, respectively. The $L_{1}$ signal component with the value $T_{2 L_{1}}^{*} \approx 100 \mu \mathrm{s}$ is characteristic of tightly bound water fraction either for lichen thalli $[13,1]$ or for many other biological systems $[14,15]$. The $L_{2}$ component with $T_{2 L_{2}}^{*} \approx 1000 \mu \mathrm{s}$, shortened by $B_{0}$ inhomogeneities, comes from water loosely bound on thallus surfaces and for higher hydration level from free water fraction (Fig. 3). The parameters for the solid component were fitted with the accuracy of

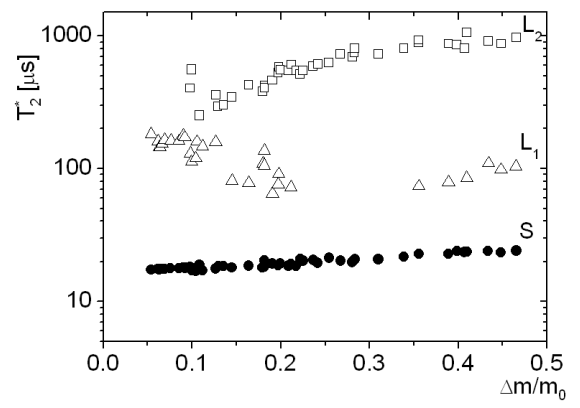

Fig. 3. The hydration dependence of proton FID relaxation times for U. antarctica. 
$2 \%$ using program CracSpin, whereas for both liquid components the accuracy of the fitted parameters is better than $10 \%$ [11].

The $T_{2 S}^{*}$ value for solid component does not change with increasing hydration level, suggesting that the structure and dynamics of thallus solid matrix is hardly modified by hydration process, thus can be used as a unit for scaling of liquid components.

The amplitude of tightly bound water signal, $L_{1} / S$, nearly saturates even for low hydration level, whereas loosely bound water signal, $L_{2} / S$, increases linearly with increasing mass, $\Delta m / m_{0}$, of water added. The hydration dependence of total liquid signal $L / S=\left(L_{1}+L_{2}\right) / S$ (Fig. 4$)$ is fitted well by the linear function

$$
L / S=(3.56 \pm 0.11) \Delta m / m_{0} \text {. }
$$

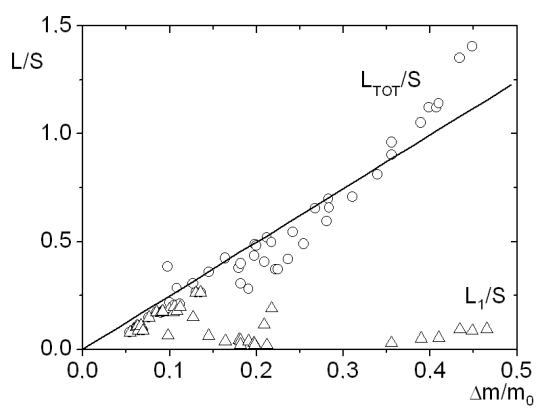

Fig. 4. The $L / S(\circ)$ and $L_{1} / S(\Delta)$ hydration dependence for $U$. antarctica.

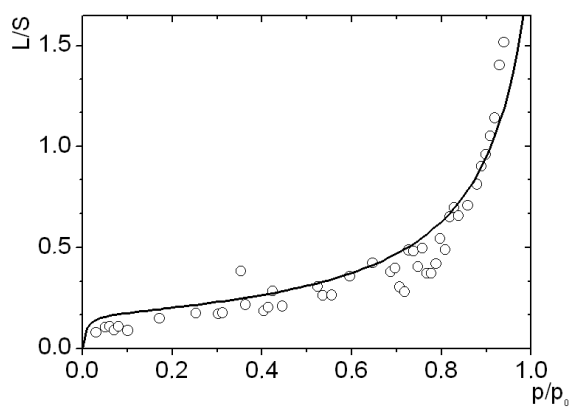

Fig. 5. Sorption isotherm fitted to total FID proton liquid component signal for water hydrating thallus of $U$. antarctica.

As the total liquid signal $L / S$ is proportional to the mass of water hydrating thallus at given air-humidity $h, L / S$ may be used to construct the NMR-isotherm, but with the shape described by the same sorption parameters $\left(\Delta M / m_{0}, b\right.$, and $\left.b_{1}\right)$ as regular sorption isotherm. Indeed, the NMR data are well fitted by the function

$$
\frac{L}{S}=A+k \frac{\Delta M}{m_{0}} \frac{b_{1} h}{(1-b h)\left(1+b_{1} h-b h\right)},
$$


where fitted parameters are solely $A$ and $k$. Best fits are obtained for $A=0$ and $k=2.45 \pm 0.06$ (see Fig. 5). The parameter $A=0$ means that the fraction of bound water "sealed" in solid matrix pores (as it is in lyophilized photosynthetic membranes) is not present in thallus of $U$. antarctica [16].

\section{Discussion}

In nearly dry $U$. antarctica thallus water differs in structurization and mobility with the proximity to the surface and thus may be grouped in, at least, three fractions: (i) very tightly bound water to primary water binding sites with elevated affinity to thallus surface (recognized by hydration courses and by sorption isotherm as water bound to primary binding sites); (ii) tightly bound water (observed by hydration courses, and distinguished by proton FID hydration dependences), presumably first hydration shell; (iii) loosely bound water. In contrast to lyophilized photosynthetic membrane structure the highly immobilized water, surrounding the manganese ions non-specifically bound on the surfaces, is not recorded [16].

\section{References}

[1] H. Harańczyk, S. Gaździński, M. Olech, New Aspects in Cryptogamic Research, Contribution in Honour of Ludger Kappen. Bibl. Lichenol. 75, 265 (2000).

[2] H. Harańczyk, J. Grandjean, M. Olech, Coll. Surf. B, Biointerfaces 28, 239 (2003).

[3] H. Harańczyk, J. Grandjean, M. Olech, M. Michalik, Coll. Surf. B, Biointerfaces 28, 251 (2003).

[4] L. Kappen, M. Breuer, M. Bölter, Polar Biology 11, 393 (1991).

[5] L. Kappen, B. Schroeter, C. Scheidegger, M. Sommerkorn, G. Hestmark, Adv. Space Res. 18, 119 (1996).

[6] M.R. Worland, W. Block, H. Oldale, Cryo-Letters 17, 31 (1996).

[7] H. Harańczyk, On Water in Extremely Dry Biological Systems, Wydawnictwo Uniwersytetu Jagiellońskiego, Kraków 2003.

[8] L. Kappen, M. Breuer, Antarctic Sci. 3, 273 (1991).

[9] B. Schroeter, M. Olech, L. Kappen, W. Heitland, Antarctic Sci. 7, 251 (1995).

[10] D.F. Gaff, Oecologia (Berl.) 31, 95 (1977).

[11] W. Wȩglarz, H. Harańczyk, J. Phys. D, Appl. Phys. 33, 1909 (2000).

[12] R.W. Dent, Textile Res. J. 47, 145 (1977).

[13] H. Harańczyk, S. Gaździński, M.A. Olech, New Phytologist 138, 191 (1998).

[14] H. Harańczyk, K. Strzałka, G. Jasiński, K. Mosna-Bojarska, Coll. Surf. A 115, 47 (1996).

[15] H. Harańczyk, W.P. Wȩglarz, S. Sojka, Holzforschung 53, 299 (1999).

[16] H. Harańczyk, A. Leja, K. Strzałka, Acta Phys. Pol. A 109, 389 (2006). 